\title{
Modulation of collagen synthesis and its gene expression in human skin fibroblasts by tocotrienol-rich fraction
}

Suzana Makpol, Faidruz Azura Jam, Yasmin Anum Mohd Yusof, Wan Zurinah Wan Ngah

Department of Biochemistry, Faculty of Medicine, National University of Malaysia, Kuala Lumpur, Malaysia

Submitted: 19 November 2010

Accepted: 21 March 2011

Arch Med Sci 2011; 7, 5: 889-895

DOI: 10.5114/aoms.2011.25567

Copyright @ 2011 Termedia \& Banach

\section{Abstract}

Introduction: Skin aging may occur as a result of increased free radicals in the body. Vitamin E, the major chain-breaking antioxidant, prevents propagation of oxidative stress, especially in biological membranes. In this study, the molecular mechanism of tocotrienol-rich fraction (TRF) in preventing oxidative stressinduced skin aging was evaluated by determining the rate of total collagen synthesis and its gene expression in human skin fibroblasts.

Material and methods: Primary culture of human skin fibroblasts was derived from circumcision foreskin of 9 to 12 year-old boys. Fibroblast cells were divided into 5 different treatment groups: untreated control, hydrogen peroxide $\left(\mathrm{H}_{2} \mathrm{O}_{2}\right)$ induced oxidative stress ( $20 \mathrm{\mu M} \mathrm{H}_{2} \mathrm{O}_{2}$ exposure for 2 weeks), TRF treatment, and pre- and post-treatment of TRF to $\mathrm{H}_{2} \mathrm{O}_{2}$-induced oxidative stress.

Results: Our results showed that $\mathrm{H}_{2} \mathrm{O}_{2}$-induced oxidative stress decreased the rate of total collagen synthesis and down-regulated $\mathrm{COL} I$ and $\mathrm{COL}$ III in skin fibroblasts. Pre-treatment of TRF protected against $\mathrm{H}_{2} \mathrm{O}_{2}$-induced oxidative stress as shown by increase in total collagen synthesis and up-regulation of $\mathrm{COL} I$ and $\mathrm{COL} \mathrm{III}(p<0.05)$ genes. However, similar protective effects against $\mathrm{H}_{2} \mathrm{O}_{2}$-induced oxidative stress were not observed in the post-treated fibroblasts.

Conclusions: Tocotrienol-rich fraction protects against $\mathrm{H}_{2} \mathrm{O}_{2}$-induced oxidative stress in human skin fibroblast culture by modulating the expression of $\mathrm{COL} I$ and COL III genes with concomitant increase in the rate of total collagen synthesis. These findings may indicate TRF protection against oxidative stressinduced skin aging.

Key words: tocotrienol-rich fraction, skin aging, collagen synthesis, gene expression.

\author{
Corresponding author: \\ Suzana Makpol PhD \\ Department of Biochemistry \\ Faculty of Medicine \\ National University \\ of Malaysia \\ Jalan Raja Muda Abdul Aziz \\ 50300 Kuala Lumpur, \\ Malaysia \\ Phone: 603-92897296, \\ 603-92897222 \\ Fax: 603-26938037 \\ E-mail: \\ suzanamakpol@yahoo.com
}

\section{Introduction}

Skin aging involves changes in skin structure with time or age. It is characterized by inability of the skin to balance its functions, finally resulting in cell death. Some changes in aging skin are dryness, wrinkles, pigmentation, lack of moisture, sagging and growth of non-cancerous or pre-cancerous cells. Skin aging may occur as a result of increased free radicals in the body [1].

Chronological skin aging is characterized by atrophy in skin due to loss of elasticity and slowing down of metabolic activity. Damage caused by the environment, especially exposure to ultraviolet radiation (photodamage), results in intrinsic aging processes such as hypertrophic repair reaction with epidermis thickening and increased melanogenesis. Other changes, such as abnormal elastic fibre aggregation, collagen 
degeneration, vasodilatation and cross-linking of blood vessels, can occur in the dermis layer of the skin. The frequent use of sun block only can allow some of the repair processes and protect against photodamage [2].

The fibroblast is a large spindle-shaped cell which is commonly present in intercellular tissue. It synthesises collagen and elastin fibres as well as extracellular matrix in areolar and dense connective tissue [3]. Fibroblasts comprise the major component of the skin and they produce and arrange extracellular matrix on the dermis as well as communicate with other cells. They play an important role in regulating skin physiology [4]. In aged skin, fibroblasts that produce collagen and elastin fibres decrease and this will result in the formation of wrinkles [5].

The major structural protein present in the dermal layer of the skin is collagen type I. It represents $90 \%$ of skin dry mass and is decreased in aging skin [6]. Small children have higher content of collagen type III. This type of collagen is present in rapidly growing tissues. When body growth is slowing down, type III collagen decreases while type I collagen increases. Meanwhile, at the age of 60 years, the level of all types of collagen in skin decrease as compared to young individuals. Overall, total collagen in skin decreases with age [7].

A major feature of aged skin is fragmentation of the dermal collagen matrix. Fragmentation results from actions of specific enzymes, i.e. matrix metalloproteinases (MMPs), which impair the structural integrity of the dermis. Fibroblasts that produce and organize the collagen matrix cannot attach to fragmented collagen. Loss of attachment prevents fibroblasts from receiving mechanical information from their support, and they collapse. In aged skin, collapsed fibroblasts produce low levels of collagen and high levels of collagendegrading enzymes (MMPs). This imbalance advances the aging process, in a self-perpetuating, never-ending deleterious cycle [8].

Oxidative stress is thought to be of primary importance in driving the aging process [9]. The free radical theory proposes that aging, including skin aging, is the cumulative result of oxidative damage to the cells and tissues of the body that arises primarily as a result of aerobic metabolism [10]. Although skin possesses extremely efficient antioxidant activities, it has been demonstrated that during aging, reactive oxygen species (ROS) levels rise and anti-oxidant defences decline.

Natural vitamin E comprises eight different forms; they are $\alpha-, \beta-, \gamma$ - and $\delta$-tocopherols and $\alpha-, \beta-, \gamma-$ and $\delta$-tocotrienols [11]. Based on its lipophilicity, vitamin $\mathrm{E}$ is considered to be the major chain-breaking antioxidant preventing the propagation of oxidative stress, especially in biological membranes [12]. Vitamin E, specifically $\alpha$-tocopherol, has been reported to be able to modulate signal transduction and gene expression in antioxidant and non-antioxidant manners [11]. Other biological functions of $\alpha$-tocopherol that are unrelated to its antioxidant properties, which include its roles in cellular signalling, gene expression, immune response and apoptosis, are now considered to be of importance [13, 14].

Recently, the different isomers of tocotrienol have gained increasing scientific interest due to their eminent antioxidant effects and a nonantioxidant activity profile that differs from tocopherols [12]. Tocotrienols are found in abundance in plant foods such as rice bran or palm oil [15]. $\alpha$-Tocotrienol has been reported to have higher antioxidant activity in liver microsome and to be able to protect membrane intrinsic protein (cytochrome $\mathrm{P}_{450}$ ) from oxidative damage compared to $\alpha$-tocopherol [16]. Thus, this study was designed to evaluate the molecular mechanism of tocotrienol, particularly tocotrienol-rich fraction, in preventing oxidative stress-induced skin aging by determining the changes in total collagen synthesis and expression of COL I, COL III and COL IV genes.

\section{Material and methods}

\section{Cell culture and treatment protocols}

This research has been approved by the Ethics Committee of the National University of Malaysia (Approval Project Code: FF-144-2007). Primary human skin fibroblasts were derived from foreskins of 9 to 12 year-old boys after circumcision. Written informed consent was obtained from parents of all subjects. The samples were aseptically collected and washed several times with 75\% alcohol and phosphate buffered saline (PBS) containing 1\% antibiotic (100 U/ml penicillin/streptomycin) (Gibco, USA) and antimycotic (100 U/ml amphotericin) (PAA, Austria) solution. After removing the epidermis, the pure dermis was cut into small pieces and transferred into a falcon tube containing $0.03 \%$ collagenase type I solution (Worthington Biochemical Corporation, USA). Pure dermis was digested in the incubator shaker at $37^{\circ} \mathrm{C}$ for $6-12 \mathrm{~h}$. Then, cells were rinsed with PBS before being cultured in Dulbecco Modified Eagle Medium (DMEM) (Flowlab ${ }^{\text {TM }}$, Australia) containing 10\% fetal bovine serum (FBS) (PAA, Austria) and 1\% antibiotic (100 U/ml penicillin/streptomycin) (Gibco, USA) and antimycotic (100 U/ml amphotericin) (PAA, Austria) solution at $37^{\circ} \mathrm{C}$ in a $5 \% \mathrm{CO}_{2}$ humidified incubator. After 5-6 days, the cultured fibroblasts were harvested by trypsinization and culture expanded into new T25 culture flasks (Nunc, Denmark) with expansion degree of $1: 4$. When the subcultures reached $80-90 \%$ confluence, serial passaging was 
done by trypsinization and the number of population doublings (PDs) was monitored. For subsequent experiments, cells were used at passage 4 (population doubling; $\mathrm{PD}<12$ ), and at passage 6 cells were exposed to $20 \mu \mathrm{M} \mathrm{H}_{2} \mathrm{O}_{2}$ for two weeks (prolonged exposure to low concentration of $\mathrm{H}_{2} \mathrm{O}_{2}$; oxidative stress-induced skin aging). As for $\mathrm{H}_{2} \mathrm{O}_{2}$-treated cells, immediately after two weeks of $\mathrm{H}_{2} \mathrm{O}_{2}$ exposure, the medium was changed to fresh DMEM for 3 days before harvesting. All cultures were harvested after attaining $80-90 \%$ confluency by cell detachment using Accutase ${ }^{\mathrm{TM}}$ (Innovative Cell Technologies, San Diego, CA).

\section{Effects of various concentrations of tocotrienol-rich fraction on cell viability}

The effects of various concentrations of TRF treatment on cell viability of human skin fibroblasts was assessed with CellTiter $96^{*}$ Aqueous NonRadioactive Cell Proliferation Assay (MTS, Promega, USA), which employs 3-(4,5-dimethylthiazol-2-yl)5-carboxymethoxyphenyl)-2-(4-sulfophenyl)-2Htetrazolium (MTS) and the electron coupling agent phenazine methosulphate (PMS). The MTS compound is reduced by the dehydrogenase enzymes found in metabolically active cells into a formazan product that is soluble in the medium. The amount of coloured formazan product is proportional to the number of viable cells.

Briefly, stock solutions of TRF Gold-Tri E 50 (Golden Hope Bioganic Sdn Bhd, Malaysia) was freshly prepared in $100 \%$ ethanol $(1: 1)$ and kept at $-20^{\circ} \mathrm{C}$ for not more than one month. Immediately before use, TRF was incubated with FBS overnight at $37^{\circ} \mathrm{C}$. Serial dilutions of TRF at concentrations of $100,200,300,400$, and $500 \mu \mathrm{g} / \mathrm{ml}$ were prepared in culture medium mixed with $50 \%$ ethanol $(1: 1)$. Cells were plated at $2 \times 10^{4}$ in a 96 -well plate and incubated overnight. Then the medium was replaced with new medium containing the various concentrations of TRF and incubated for $24 \mathrm{~h}$ at $37^{\circ} \mathrm{C}$, in $5 \% \mathrm{CO}_{2}$. After incubation, $20 \mu \mathrm{MTS}$ was added and cells were further incubated for $2 \mathrm{~h}$. The absorbance of MTS formazan formed was measured at $490 \mathrm{~nm}$ with a microtitre plate reader (VeraMax Molecular Devices, USA). The viability assay was performed to obtain the optimum dose of TRF treatment for subsequent experiments. In the subsequent experiments, TRF-treated human skin fibroblasts were incubated with $500 \mu \mathrm{g} / \mathrm{ml} \mathrm{TRF}$ before or after the $\mathrm{H}_{2} \mathrm{O}_{2}$-induced oxidative stress while untreated cells were cultured in Dulbecco Modified Eagle Medium (DMEM) containing 10\% fetal bovine serum (FBS) (PAA, Austria). The medium for the untreated cells was changed in parallel to the treated cells. Both untreated and treated cells were harvested on the same day.

\section{Determination of total collagen}

Total collagen was determined by the picro-sirius method using Sircol assay kit (Biocolor, UK). The principle of this method was based on binding of picro-sirius red dye to total collagen synthesised in the cell, which can be measured using a spectrophotometer at $540 \mathrm{~nm}$. Briefly, Sircol dye reagent was added to each Eppendorf tube filled with blank solution, sample and standard collagen. Colour changes were measured using a spectrophotometer at $540 \mathrm{~nm}$ and total collagen was calculated based on a plotted collagen standard curve.

\section{Total RNA extraction}

Total RNA from human skin fibroblasts in different groups was extracted using TRI Reagent (Molecular Research Center, Cincinnati, $\mathrm{OH}$ ) according to the manufacturer's instructions. Polyacryl Carrier (Molecular Research Center, Inc.) was added in each extraction to precipitate the total RNA. The extracted total RNA pellet was then washed with $75 \%$ ethanol and air-dried before being dissolved in RNase and DNase free distilled water. Total RNA was stored at $-80^{\circ} \mathrm{C}$ immediately after extraction. Yield and purity of the extracted total RNA were determined by Nanodrop (Thermo Scientific, USA).

\section{Primer design and quantitative real-time RT-PCR}

The primer sequences for GAPDH, COL I, COL III and COL IV were referred to the GenBank (http://www.ncbi.nlm.nih.gov) database and were designed using Primer 3 software (http://frodo.wi. mit.edu/primer3) [17] (Table I). The targets amplified by the primer pairs were characterised by BLAST (Basic Local Alignment Search Tool; http://blast.

Table I. Sequences of forward and reverse primers and the product size

\begin{tabular}{|lccc|}
\hline Target gene & Forward primer (5'-3') & Reverse primer (5'-3') & Product size [bp] \\
\hline GAPDH & CTTTGGTATCGTGGAAGGACTC & GTAGAGGCAGGGATGATGTTCT & 217 \\
\hline COLI & GTGCTAAAGGTGCCAATGGT & ACCAGGTTCACCGCTGTTAC & 216 \\
\hline COL III & CCAGGAGCTAACGGTCTCAG & CAGGGTTTCCATCTCTTCCA & 103 \\
\hline COL IV & CTGGTCCAAGAGGATTTCCA & TCATTGCCTTGCACGTAGAG & 193 \\
\hline
\end{tabular}


ncbi.nlm.nih.gov). These genes were amplified using Bio-Rad iCycler and iScript ${ }^{\text {TM }}$ One-Step RT-PCR Kit with SYBR ${ }^{\circledR}$ Green (Biorad, USA) to a final volume of $25 \mu \mathrm{l}$ to determine the specificity of the primers. The size of the PCR products was then checked by running on $1.8 \%$ agarose gel pre-stained with ethidium bromide along with a 100 bp DNA step ladder (Promega). Optimization of the real-time RT-PCR procedures was established by running the standard curve. Four serial dilutions of total RNA were used ranging from neat, 2, 4, 8 and 16 fold. The amplification programme was as follows: CDNA synthesis at $50^{\circ} \mathrm{C}$ for $30 \mathrm{~min}$, iscript reverse transcriptase inactivation at $94^{\circ} \mathrm{C}$ for $2 \mathrm{~min}$, followed by 38 amplification cycles of denaturation at $94^{\circ} \mathrm{C}$ for $30 \mathrm{~s}$ and $60^{\circ} \mathrm{C}$ (primer annealing and extension) for $30 \mathrm{~s}$. After the end of the last cycle, melting curve analysis was generated at $95^{\circ} \mathrm{C}$ for $1 \mathrm{~min}, 55^{\circ} \mathrm{C}$ for $1 \mathrm{~min}$ and $60^{\circ} \mathrm{C}$ for $10 \mathrm{~s}$ (70 cycles, set point temperature after cycle 2 increase by $0.5^{\circ} \mathrm{C}$ ). Real time RT-PCR was performed with the glyceraldehyde 3-phosphate dehydrogenase (GAPDH) gene used as an internal reference to normalize all RNA expression levels [18].

\section{Agarose gel electrophoresis}

$10 \mu \mathrm{l}$ of PCR product from each sample was added to $2 \mu \mathrm{l}$ of loading dye and pipetted into the well. $2 \mu \mathrm{l}$ of 100 bp DNA ladder was pipetted into the first well as a marker. TBE $1 \mathrm{X}$ buffer was added to prepare the ionic condition which allows DNA migration. Electrophoresis was run at $110 \mathrm{~V}$ for $1 \mathrm{~h}$ and the presence of a band was observed using the gel documentation system FlourChem FC2 (Alpha Innotech).

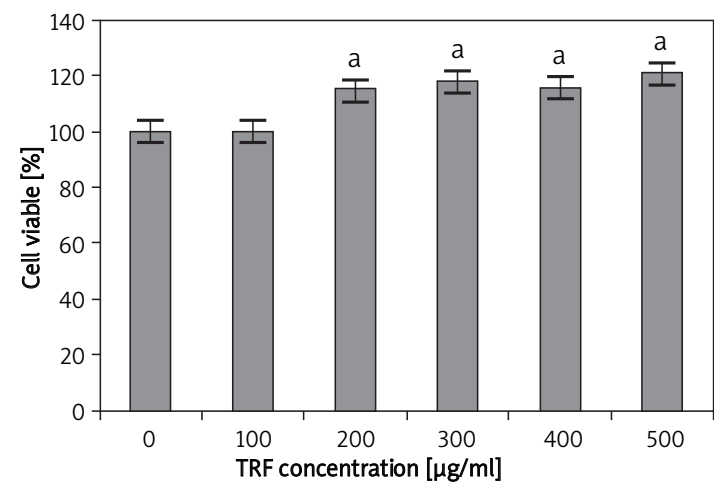

Figure 1. Effects of various concentrations of tocotrienol-rich fraction (TRF) on skin fibroblasts after incubation for $24 \mathrm{~h}$ at $37^{\circ} \mathrm{C}$. Incubation with TRF caused a significant increase in the number of viable cells for skin fibroblasts. The percentage of viable cells was significantly increased at TRF concentrations of 200, 300, 400 and $500 \mu \mathrm{g} / \mathrm{ml}$. Correlation analysis showed a significant correlation between cell viability and concentration of $\operatorname{TRF}\left(R^{2}=0.865\right.$, $p=0.026)$

ap $<0.05$ compared to control, data are presented as mean $\pm S D$

\section{Statistical analysis}

Experiments were performed at least three times and data were analysed by Student's $t$-test and one-way analysis of variance (ANOVA). Significance was accepted at $p<0.05$.

\section{Results}

Incubation with increasing concentrations of TRF caused a significant increase in the number of viable cells for skin fibroblasts. The percentage of viable cells was significantly increased at TRF concentrations of 200,300, 400 and $500 \mu \mathrm{g} / \mathrm{ml}$ $(p<0.05)$ (Figure 1). Correlation analysis showed a significant correlation between cell viability and concentration of TRF $\left(R^{2}=0.865, p=0.026\right)$.

Comparison of total collagen synthesised in different treatment groups of skin fibroblasts showed that total collagen was increased in TRF-treated fibroblasts compared to control $(p<0.05)$ (Figure 2). $\mathrm{H}_{2} \mathrm{O}_{2}$-induced oxidative stress caused a significant reduction in the synthesis of total collagen. Pre-treatment of TRF to $\mathrm{H}_{2} \mathrm{O}_{2}-$ induced oxidative stress however increased the synthesis of total collagen as compared to untreated control and $\mathrm{H}_{2} \mathrm{O}_{2}$-treated fibroblasts ( $p<0.05$ ). Post-treatment of TRF to $\mathrm{H}_{2} \mathrm{O}_{2}$-induced oxidative stress did not produce similar protective effects against $\mathrm{H}_{2} \mathrm{O}_{2}$-induced oxidative stress.

Agarose gel electrophoresis showed that each PCR product appeared as a single band (Figure $3 \mathrm{~A}$ ), while the melting curve analysis showed a single narrow peak of each PCR product (Figures $3 B$ and 3C) without the formation of a primer-dimer

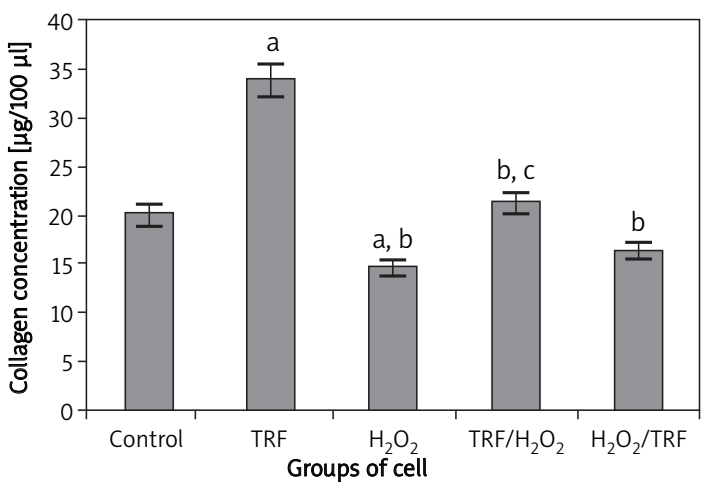

Figure 2. Comparison of total collagen synthesised in different treatment groups of skin fibroblasts. Total collagen was increased in TRF-treated fibroblasts while $\mathrm{H}_{2} \mathrm{O}_{2}$-induced oxidative stress caused a reduction in the synthesis of total collagen. Pretreatment of TRF to $\mathrm{H}_{2} \mathrm{O}_{2}$-induced oxidative stress increased the synthesis of total collagen $a_{p}<0.05$ compared to untreated control, $b p<0.05$ compared to TRF-treated fibroblasts, $c p<0.05$ compared to $\mathrm{H}_{2} \mathrm{O}_{2}$-induced oxidative stress; data are presented as mean $\pm S D$ 

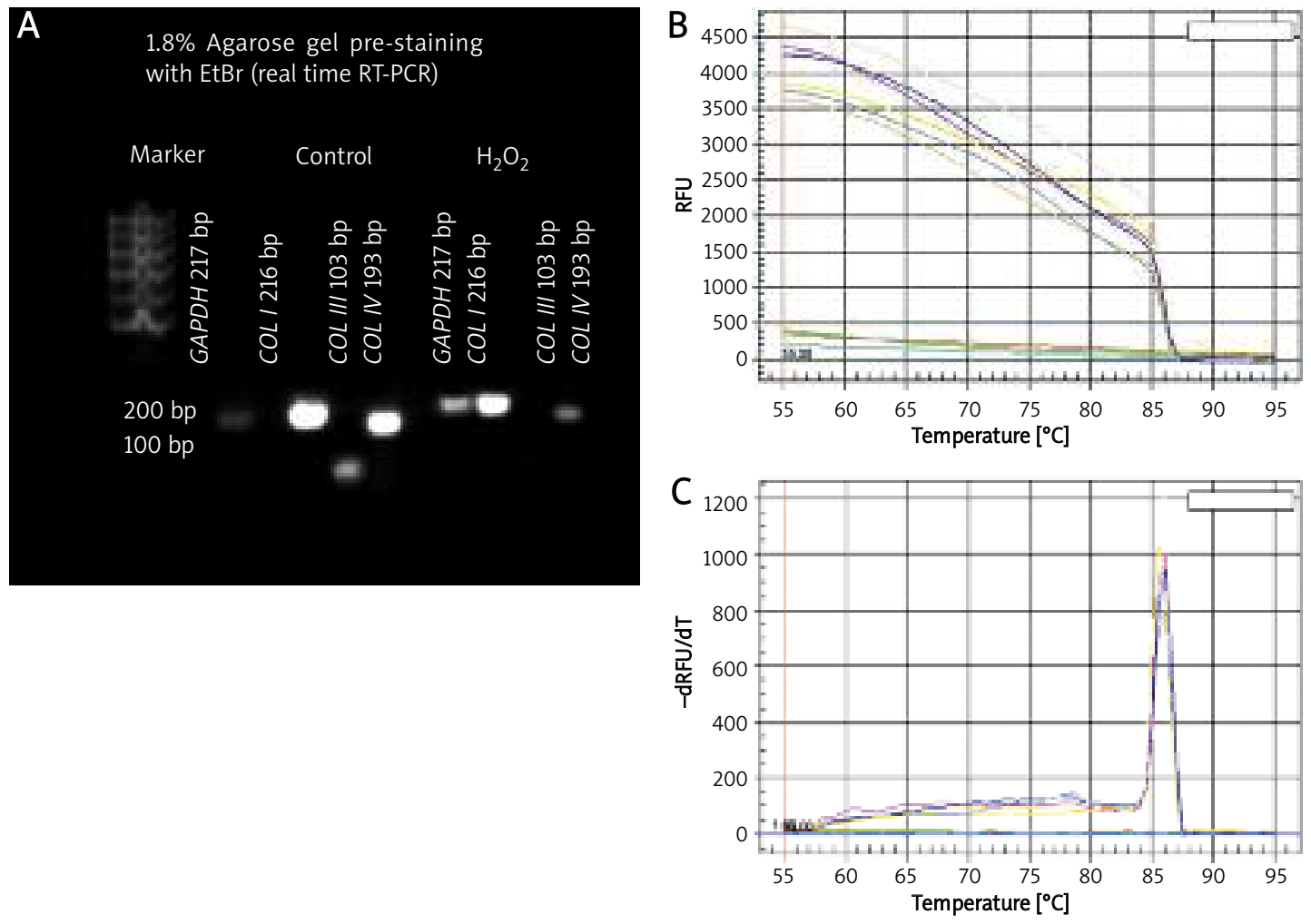

Figure 3. Agarose gel electrophoresis showed that each PCR product appeared as a single band (A) while the melt curve (B) and melt peak (C) analysis showed a single narrow peak of each PCR product without the formation of a primer-dimer structure, indicating that the designed primers and the real-time RT-PCR protocols were specific

structure, indicating that the primers designed and the real-time RT-PCR protocols were specific.

COL I and COL III were down-regulated in skin fibroblasts which were exposed to $\mathrm{H}_{2} \mathrm{O}_{2}$-induced oxidative stress compared to untreated control fibroblasts $(p<0.05)$ (Figures 4 and 5). Pre-treatment

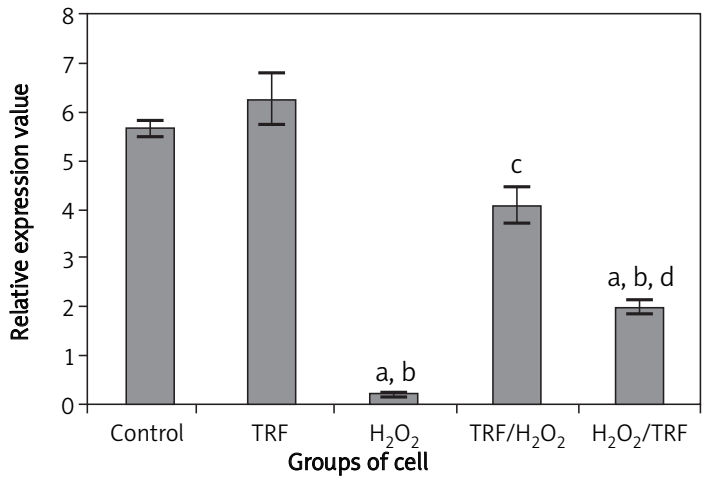

Figure 4. Expression of $\mathrm{COL} /$ in different treatment groups of skin fibroblasts. COL I was down-regulated in skin fibroblasts with $\mathrm{H}_{2} \mathrm{O}_{2}$-induced oxidative stress. Pre-treatment of TRF to $\mathrm{H}_{2} \mathrm{O}_{2}$-induced oxidative stress increased the expression of $\mathrm{COL} /$ gene $a p<0.05$ compared to untreated control, $b p<0.05$ compared to TRF-treated fibroblasts, $c p<0.05$ compared to $\mathrm{H}_{2} \mathrm{O}_{2}$-induced oxidative stress, $d p<0.05$ compared to TRF pre-treated fibroblasts, data are presented as mean $\pm S D$ of TRF to $\mathrm{H}_{2} \mathrm{O}_{2}$-induced oxidative stress increased the expression of $\mathrm{COL} /$ and $\mathrm{COL}$ III genes as compared to $\mathrm{H}_{2} \mathrm{O}_{2}$-treated fibroblasts $(p<0.05)$. Post-treatment of TRF to $\mathrm{H}_{2} \mathrm{O}_{2}$-induced oxidative stress did not produce similar protective effects against $\mathrm{H}_{2} \mathrm{O}_{2}$-induced oxidative stress as both $\mathrm{COL} /$ and $\mathrm{COL}$ III were down-

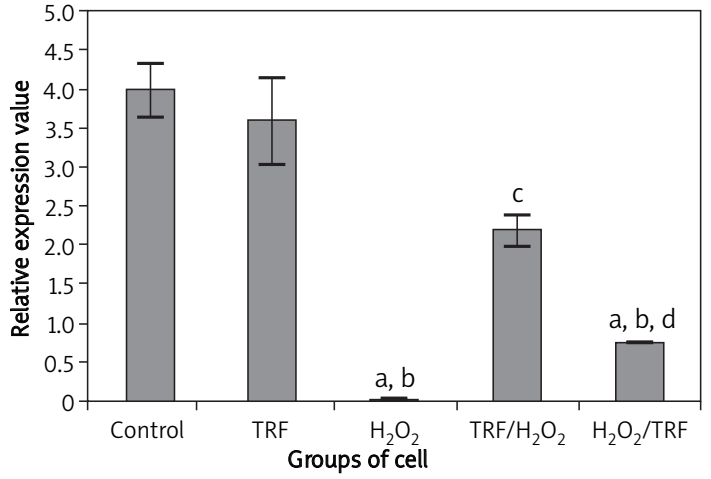

Figure 5. Expression of $\mathrm{COL} I / I$ in different treatment groups of skin fibroblasts. COL III was downregulated in skin fibroblasts with $\mathrm{H}_{2} \mathrm{O}_{2}$-induced oxidative stress. Pre-treatment of TRF to $\mathrm{H}_{2} \mathrm{O}_{2}$-induced oxidative stress increased the expression of COL III gene

$a_{p}<0.05$ compared to untreated control, $b_{p}<0.05$ compared to TRF-treated fibroblasts, $c p<0.05$ compared to $\mathrm{H}_{2} \mathrm{O}_{2-}$ induced oxidative stress, $d p<0.05$ compared to TRF pretreated fibroblasts, data are presented as mean $\pm S D$ 


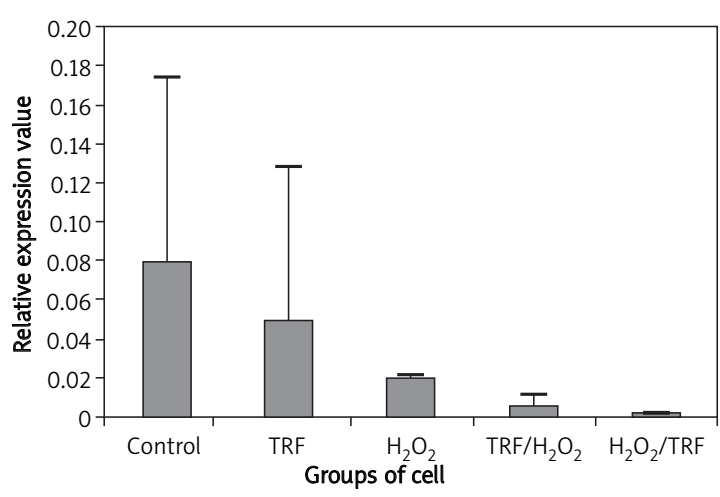

Figure 6. Expression of COL $N$ in different treatment groups of skin fibroblasts

Data are presented as mean $\pm S D$

regulated as compared to control. No significant results were observed for COL $N$ (Figure 6).

\section{Discussion}

This study explored the molecular mechanism of TRF in preventing oxidative stress-induced skin aging. Oxidative stress and the accumulation of intracellular reactive oxygen species (ROS) play an important role in the induction of cellular aging. The in vitro life span of cells was reported to be related to the redox state of the microenvironment, suggesting that cellular aging can be induced or accelerated by conditions and agents that increase the oxidative stress and the production of ROS [19].

Our results showed that exposure to a prolonged low dose of $\mathrm{H}_{2} \mathrm{O}_{2}$ decreased the expression of $\mathrm{COL} I$ and $\mathrm{COL}$ III genes with concomitant reduction in total collagen synthesis. A previous study reported that senescent or aged fibroblasts had decreased expression of several extracellular matrix components such as collagen I- $1 \alpha$, collagen III- $1 \alpha$ and elastin besides increased expression of collagenase and stromelysin - two enzymes that serve to break down the extracellular matrix [8]. These reports supported our data showing that total collagen synthesis decreased with down-regulation of $\mathrm{COL} I$ and COL III genes in oxidative stress-induced senescent fibroblasts. It has also been reported that among the changes in gene expression in senescent or aged cells, the over-expression of matrix metalloproteinases (MMPs) was striking, resulting in loss of proteins which maintained the ultrastructural shape. Relative overproduction of collagenase in aging cells was proposed as the matrix-degrading phenotype of senescent cells [20]. Another study reported that a prolonged low dose of $\mathrm{H}_{2} \mathrm{O}_{2}$ treatment not only induced irreversible cell cycle arrest and senescent-like morphology, but also caused a sustained elevated level of DNA damage which ultimately leads to aging [21].

In this study, TRF seems to provide protection against $\mathrm{H}_{2} \mathrm{O}_{2}$-induced oxidative stress which may cause skin aging. Pre-treatment of TRF to oxidative stress-induced cellular aging upregulated $\mathrm{COL} /$ and COL III with a concomitant increase in total collagen synthesis. These effects can be attributed to its antioxidant properties. A previous study reported that antioxidant has been proven to give protection to the skin against free radical damage. Thus one strategy to increase protection against oxidative stress in the skin is by supporting the antioxidant endogenous system [22].

Tocotrienol-rich fraction contains both tocotrienol and tocopherol. Therefore the observed effects of TRF in this study in protecting against $\mathrm{H}_{2} \mathrm{O}_{2}$-induced oxidative stress may also be partly due to tocopherol and not tocotrienol alone. A comparative study on the action of tocopherols and tocotrienols as antioxidants showed that both tocopherols and tocotrienols exerted the same reactivities toward radicals and the same antioxidant activities against lipid peroxidation in solution and liposomal membranes, besides similar mobilities within the cell membranes. Tocotrienols however were more readily transferred between the membranes and incorporated into the membranes than tocopherols [23], while another study reported that tocotrienol was easily absorbed by the skin and suitable to be used as vitamin E cream [24].

In this study, pre-treatment of TRF to oxidative stress-induced skin aging was found to be more beneficial than post-treatment. This may be explained by damage to DNA caused by oxidative stress which already occurred before the cells were treated with TRF in the post-treated group. Oxidative stress has been reported to cause DNA damage which increased significantly in senescent cells, especially in post-mitotic tissues, and that the levels correlated with aging and age-related diseases [25]. Tahara et al. reported that age-related increase in the levels of oxidative DNA base damage was a significant contributor to many agerelated pathological diseases. If accumulation of damaged DNA exceeds its elimination by the DNA repair mechanism, cellular senescence or apoptosis takes place and contributes to the aging process [26]. Our data suggested that TRF may prevent oxidative stress-induced skin aging but did not exert its protective effects when treatment was started after oxidative stress induction, as damage to DNA had already occurred.

In conclusion, TRF may protect against oxidative stress-induced skin aging by modulating the expression of COL I and COL III genes with a concomitant increase in the rate of collagen synthesis. These protective effects however were only observed in the pre-treated fibroblasts.

\section{Acknowledgments}

This study was funded by the Ministry of Higher Learning under the Fundamental Research Grant 
Scheme UKM-FF-03-FRGS0001-2006 and National University of Malaysia FF-152-2010.

\section{References}

1. Chandrasoma P, Taylor CR. Concise pathology. $3^{\text {rd }}$ ed. Singapore. Appleton \& Lange 2001.

2. Gilchrest BA. A review of skin ageing and its medical therapy. Br J Dermatol 2007; 135: 867-75.

3. Tortora GJ, Derrickson SR. Principle of anatomy and physiology. United States of America. John Wiley \& Sons, Inc. 2006.

4. Werner S, Smola H. Paracrine regulation of keratinocyte proliferation and differentiation. Trends Cell Biol 2001; 11: 143-6.

5. Varani J. Decreased collagen production in chronologically aged skin. Arch Dermatol 2006; 168: 1861-8.

6. Frank W, Luis AG, Sewon K, et al. In vivo stimulation of de novo collagen production caused by cross-linked hyaluronic acid dermal filler injections in photodamaged human skin. Arch Dermatol 2007; 143: 155-63.

7. Ramos SM, Carneiro DS. Elderly skin and its rejuvenation: products and procedures for the aging skin. J Cosmet Dermatol 2007; 6: 40-50.

8. Fisher GJ, Varani J, Voorhees JJ. Looking older: fibroblast collapse and therapeutic implications. Arch Dermatol 2008; 144: 666-728.

9. Feher J, Nemeth E, Nagy V, Lengyel G, Feher J. The preventive role of coenzyme Q10 and other antioxidants in injuries caused by oxidative stress. Arch Med Sci 2007; 3: 305-14.

10. Harman D. Aging: phenomena and theories. Ann New York Acad Sci 1998; 854: 1-7.

11. Zingg JM. Modulation of signal transduction by vitamin $\mathrm{E}$. Mol Aspects Med 2007; 28: 481-506.

12. Sebastian S, Muller WE, Eckert GP. Tocotrienols: constitutional effects in aging and disease. J Nutr 2005; 135: 151-4.

13. Azzi A, Ricciarelli R, Zingg JM. Non-antioxidant molecular functions of $\alpha$-tocopherol (vitamin E). FEBS Lett 2002; 519: 8-10.

14. Brigelius-Flohe R, Kelly FJ, Salonen JT, et al. The European perspective on vitamin E: current knowledge and future research. Am J Clin Nutr 2002; 76: 703-16.

15. Theriault A, Chao JT, Wang Q, et al. Tocotrienol: a review of its therapeutic potential. Clin Biochem 1999; 32: 309-19.

16. Mutalib MSA, Huzwah KA, Wahle KWJ. Palm tocotrienol rich fraction (TRF) ia a more effective inhibitor of LDL oxidation and endothelial cell lipid peroxidation than alpha-tocopherol in vitro. Food Res Int 2003; 36: 405-13.

17. Rozen S; Skaletzky HJ. Primer3 on the WWW for general users and for biologist programmers. In: Bioinformatics methods and protocols: methods in molecular biology. Krawetz S, Misener S(eds.), Humana Press, Totowa, NJ, USA 2000; 365-86.

18. Zainuddin A, Chua KH, Abdul Rahim N, Makpol S. Effects of experimental treatment on GAPDH mRNA expression as a housekeeping gene in human diploid fibroblasts. BMC Mol Biol 2010; 11: 59.

19. Droge W. Free radicals in physiological control of cell function. Physiol Rev 2002; 82: 47-95.

20. Mawal-Dewan M, Lorenzini A, Frisoni L, Zhang H, Cristofalo VJ, Sell C. Regulation of collagenase expression during replicative senescence in human fibroblasts by Akt-forkhead signaling. J Biol Chem 2002; 277: 7857-64.
21. Di Micco R, Cicalese A, Fumagalli M, et al. DNA damage response activation in mouse embryonic fibroblasts undergoing replicative senescence and following spontaneous immortalization. Cell Cycle 2008; 7: 3601-6.

22. Branka R, Mirjana G, Estelle TT, Fabrice P, Francoise F. Simultaneous absorption of vitamins $C$ and $E$ from topical micro emulsions using reconstructed human epidermis as a skin model. Eur J Pharm Biopharm 2008; 72: 69-75.

23. YoshidaY, Niki E, Noguchi NC. Comparative study on the action of tocopherols and tocotrienols as an antioxidant: chemical and physical effects. Chem Physic Lipids 2003; 123: 63-75.

24. Packer L, Weber SU, Rimbach G. Molecular aspects of alpha-tocotrienol antioxidant action and cell signaling. J Nutr 2001; 131: 3175-81.

25. Bayens JW. The Maillard hypothesis on aging: time to focus on DNA. Ann New York Acad Sci 2002; 959: 360-7.

26. Tahara S, Matsuo M, Kaneko T. Age-related changes in oxidative damage to lipids and DNA in rat skin. Mech Ageing Dev 2001; 122: 415-26. 